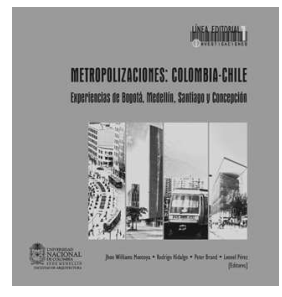

\title{
Jhon Williams Montoya, Rodrigo Hidalgo, Peter Brand y Leonel Pérez (editores). Metropolizaciones Colombia - Chile. Experiencia de Bogotá, Medellín, Santiago y Concepción
}

\author{
Medellín: Facultad de Arquitectura, Universidad Nacional de \\ Colombia, 2014. 324 p. \\ ISBN: 978-958-775-173-4
}

Ángela Milena Alzate Navarro ${ }^{1}$

\begin{abstract}
Este libro es el resultado de una alianza entre grupos de investigación de la Universidad Nacional de Colombia -Grupo de Estudios sobre la Problemática Urbano-Regional de Colombia (Geourbe) del Departamento de Geografía, Sede Bogotá y Grupo Dinámicas Urbano-Regionales de la Escuela de Planeación Urbano-Regional de la Facultad de Arquitectura, Sede Medellín-, que se afianzó a través del proyecto Geografía de la Planificación Metropolitana, e incluyó el desarrollo en conjunto con la Pontificia Universidad Católica de Chile, del "Seminario Dinámicas Metropolitanas Colombia-Chile, diálogo de Bogotá, Medellín, Santiago y Concepción", realizado en Santiago de Chile entre los días 26 y 27 de noviembre de 2012, con la participación, además, de varios investigadores de la Universidad de Concepción. Los trabajos presentados en el seminario conformaron las bases teóricas y metodológicas para abrir el debate frente a la realidad territorial contemporánea de cuatro ciudades de Chile y Colombia inscritas bajo nuevas geografías de reestructuración metropolitana y regional: Santiago de Chile y Bogotá como centros político-económicos dominantes en la escala nacional y representativos de estas dinámicas en América Latina; y Concepción y Medellín
\end{abstract}

Escuela de Planeación Urbano-Regional, Universidad Nacional de Colombia, Sede Medellín (Colombia).E-mail: amalzat1@unal.edu.co como ciudades medianas con fuertes implicaciones en los procesos de reestructuración socioeconómica y territorial regionales.

"Metrópolis en definición: hacia una geografía comparada entre Colombia y Chi$l^{\prime \prime}$ con esta frase que sugiere dinamismo -reestructuración territorial y similitudes y/o correspondencias en las formas de urbanización entre estos dos países-, los editores introducen la problemática contemporánea de los procesos de metropolización en curso, las tendencias y los retos en los casos estudiados, y los prioridades en la agenda de investigación urbana sobre las metrópolis. El fenómeno de la metropolización se reconoce en una escala supranacional, bajo las lógicas globales del capitalismo, pero con una reciente reconsideración de la importancia de la escala nacional, es decir, el papel del Estado-Nación en la formación del marco jurídico e institucional que fundamenta los procesos de reestructuración metropolitana.

El libro se estructura a partir de cuatro ejes temáticos que giran en torno a las tendencias, problemáticas y apuestas en materia de metropolización en las cuatro ciudades estudiadas de Colombia y Chile. La primera parte, metropolización y nuevas formas urbano-regionales, aborda las tendencias metropolitanas de incorporación en economías nacionales globalizadas en expansión, partiendo de los procesos de reestructuración 
política y económica acontecidos durante las últimas tres décadas, como parte de los cambios en la economía internacional de la fase posfordista del capitalismo global; y luego, estudia los correlatos de estos cambios asociados con la morfología y las formas de crecimiento urbano-regional de tres casos: Santiago y Concepción en Chile, y Bogotá en Colombia.

Esta primera parte del libro se organiza a partir de cinco textos, el primero toma como caso el proceso de metropolización de la Macrozona Urbana Central Chilena (MZUC), los autores -Hidalgo, Arenas, Sánchez y Volker- estudian las formas tipológicas de crecimiento -edificatorias, infraestructurales y de equipamientos- que expresan territorialmente la configuración espacial metropolitana contemporánea del mayor proceso de urbanización de Chile; luego, explican las correlaciones territoriales de esta nueva morfología con la modificación y complejización de la vulnerabilidad y la sustentabilidad (Sánchez, 2010) de esta zona del país, para finalizar con un análisis frente a los retos que esta situación implica para el ordenamiento, la gestión y la administración urbana y regional de Chile. El segundo, desde la perspectiva del desarrollo urbano, aborda las nuevas geografías socioespaciales en Santiago de Chile en el periodo comprendido entre 1992 y 2012, su autor, Fuentes, estudia los efectos socioespaciales de las tendencias de crecimiento metropolitano tanto las tendencias tradicionales expansivas y dispersas de las comunas periféricas como a través de expresiones espaciales recientes de verticalización o densificación de las comunas centrales; estas transformaciones están directamente relacionadas con los procesos de cambio socioeconómico y socioprofesional que hacen parte de la reestructuración política y económica introducidas por la ideología neoliberal. El tercer texto, estudia la dinámica de expansión urbana del Área Metropolitana de Concepción, sus autores -Rojas, Plata, Valdebenito, Muñiz y De la Fuente- utilizan el Modelo de Regresión Geográficamente Ponderada (RGP) para modelar espacialmente la dinámica del crecimiento metropolitano de Concepción entre 2001 y 2009 utilizando factores geográficos de tres tipos -socioeconómicos, biofísicos y de planificación-, para luego explicar a través de los resultados, los factores más significativos que influyen en el crecimiento metropolitano de manera diferencial en cada comuna. El cuarto texto aborda las implicaciones de la globalización y el neoliberalismo en la forma urbana de Bogotá durante la fase posindustrial; revisa la idea de ciudad posindustrial, estudia las transformaciones demográficas y morfológicas de las dos últimas décadas en Bogotá, evalúa los cambios recientes sucedidos en la economía urbana relativos a la desindustrialización y la tercerización, y estudia la morfología física y social como correlato de estos cambios; Montoya concluye con algunas reflexiones frente a la ecología social actual de Bogotá como resultado de las prácticas demográficas, políticas, económicas y físicas de las últimas décadas. El quinto y último texto de la primera parte del libro, estudia las transformaciones residenciales en la periferia metropolitana norte de Bogotá entre 1990 y 2010, enfatizando el análisis en los barrios cerrados en el contexto de metropolización; el autor, Santana, propone una discusión teórica entre la globalización y la metropolización, el papel de los barrios cerrados en este contexto, luego propone una clasificación de conjuntos cerrados adaptada a la periferia metropolitana de Bogotá; y finalmente, valiéndose de dos categorías propone estudiar el fenómeno de los barrios cerrados: las precariópolis y la privatópolis (Hidalgo et al., 2008) presentando algunos de los aspectos más relevantes de la producción de los barrios cerrados vinculados con las transformaciones introducidas por la globalización y las políticas neoliberales.

La segunda parte del libro, aborda la temática correspondiente al medio ambiente y las ruralidades metropolitanas; es decir, las relaciones entre los procesos propios de la naturaleza con las demandas ambientales y ecosistémicas, y las transformaciones territoriales de los entornos rurales introducidas por la expansión de la urbanización metropolitana. Esta parte del libro se organiza a partir de cinco textos; el primero, se aproxima a la ruralidad metropolitana y a la diversidad social implícita en estas nuevas formas de espacio rural, desde la reflexión frente a las tendencias periurbanas rurales de la Región Metropolitana de Santiago (RMS); los autores -Salazar-Burrows, Montoya y Osses- introducen a la reflexión partiendo de dos conceptos: urbanidad difusa y ruralidad metropolita- 
na; además, ponen en contexto los procesos de transformación socioespacial y expansión periurbana de la RMS, que luego se validan a través de una metodología que involucra categorías funcionales de la ruralidad y categorías socioeconómicas y socioprofesionales que expresan y explican las transformaciones recientes de la región extendida y sus implicaciones para la sustentabilidad territorial de escala metropolitana y regional. El segundo texto, desde una perspectiva relacional entre naturaleza y cultura, presenta un análisis de la península de Hualpén en el Área Metropolitana de Concepción (AMC) y su relación con la sustentabilidad de los procesos urbanos; los autores -Muñoz, Aguayo y Azócar- a través de una argumentación teórica y empírica, muestran la importancia ecológica, ambiental, histórica y patrimonial de la península para el AMC, y la necesidad de proteger y gestionar estos espacios naturales como parte de las funciones territoriales vitales para la sustentabilidad y la calidad de vida metropolitana. El tercer texto, estudia la importancia de los bordes de agua como espacios públicos y elementos estructurantes urbanos tomando como caso de estudio el Área Metropolitana de Concepción, los autores -Muñoz y Pérez- muestran el papel de los bordes de agua en la evolución urbana y metropolitana del AMC, y los procesos de planificación asociados a estos; finalmente, se presentan algunas consideraciones frente a los retos de la planificación urbana, el ordenamiento territorial y la gestión de los bordes de agua en el sistema de espacialidades públicas metropolitanas. El cuarto, parte de una discusión teórica que incorpora la ruralidad metropolitana como una categoría geográfica y analítica apropiada para describir y explicar las dinámicas contemporáneas de la ruralidad en entornos metropolitanos y las conexiones territoriales que las antecedieron, como lo denomina Agudelo, su autor, un palimpsesto territorial, es decir, una reescritura de las dinámicas territoriales rurales actuales fuertemente influenciadas por la expansión y dispersión de la urbanización de las áreas metropolitanas cercanas, sobre los usos agrarios tradicionales que aún persisten; esto conlleva a una complejización y heterogenización de los procesos rurales, para ello, toma como caso el entorno metropolitano de la ciudad de Medellín. El quinto texto de la segunda parte del libro, estudia las transfor- maciones del verde urbano en la planeación de Medellín, el autor, Vélez, estructura su argumentación a partir de dos tendencias de incorporación de las áreas verdes en la planeación: "fragmentación, residualidad y confinamiento como rasgos dominantes" en la conformación del espacio verde urbano de Medellín durante el siglo XX, y "linealidad, tematización y periferización de los grandes espacios verdes" (p. 166 y 168); concluye con una reflexión frente al futuro de los espacios verdes y las posibles perspectivas teóricas y técnicas que lo orientarán.

La tercera parte del libro desarrolla el tema de la movilidad metropolitana, se estructura a partir de tres textos tomando como casos de estudio grandes proyectos o procesos urbanos asociados a la movilidad de Santiago, Concepción y Medellín respectivamente. El primero, examina la implementación del sistema de transporte Transantiago desde un enfoque de movilidad urbana; la autora, Jirón, analiza los principales problemas del transporte en Santiago, las implicaciones territoriales y el modelo de gestión del Plan Transantiago, los componentes de este: red de transporte, infraestructura, administrador financiero y sistema de información; concluye con algunas consideraciones frente a las dificultades conceptuales, sociales, económicas y políticas que acompañaron la ejecución del plan y sus consecuencias territoriales. El segundo texto, desde una perspectiva constructivista y valiéndose de metodologías etnográficas, afronta el desafío de recoger de los transeúntes, las voces y vivencias del sentido de lo metropolitano en Concepción, una forma de abordar la metropolización diferente y alternativa a las metodologías estadísticas y cuantitativas sofisticadas, pero que adquiere validez por la legitimación y sentido de inclusión social; sus autores -Ganter y Herrera- logran identificar los sentimientos de orgullo e inconformidad que hacen parte de la cotidianidad y de la construcción social del sentido metropolitano de Concepción. El tercer texto, estudia el impacto en la movilidad de los sectores populares de Medellín, de los cables aéreos construidos desde el año 2004; el autor de este trabajo, Brand, analiza el significado social que tiene la movilidad, contextualiza y caracteriza los Metrocables en Medellín, examina los impactos sociales de su implementación, y explica las resonancias 
políticas que movilizan este tipo de proyectos urbanos en un contexto de movilidad urbana y metropolitana cada vez más complejo.

La cuarta y última parte del libro, desarroIla una temática de creciente interés asociada a la gobernanza y gestión metropolitana del suelo. Esta parte se estructura a partir de cuatro textos; el primero, desarrolla una reflexión frente a los desafíos de sustentabilidad y gobernanza para el desarrollo urbano chileno en la planificación territorial de los espacios metropolitanos; sus autores -Arenas e $\mathrm{Hi}$ dalgo- examinan las principales dificultades para constituir mecanismos o instrumentos de gestión territorial metropolitana, basados en el análisis de la complejidad institucionalidad que fundamenta los marcos regulatorios del ordenamiento territorial chileno, y en el modelo disperso y fragmentado de las áreas metropolitanas del país; concluyen con algunos de los principales desafíos frente a la ordenación y gestión metropolitana. El segundo texto, estudia la gobernanza metropolitana en Bogotá y las dificultades de la integración regional, su autora, Duque, primero aborda el tema desde una perspectiva teórica poniendo en tensión los debates actuales en torno a este tema y la urgencia que reviste en el actual contexto político y económico, luego hace una revisión histórica de los intentos de integración regional de Bogotá y Cundinamarca, para después concentrar el examen de la Región Capital Bogotá-Cundinamarca analizando el modelo de gobernanza, las oportunidades políticas en torno a este, los actores con sus prácticas y modos de gestión, la institucionalidad y las perspectivas frente al futuro de la integración a escala regional de esta zona del país. El tercer texto, estudia los grandes proyectos urbanos en Colombia como forma creciente de la intervención urbana; la autora, Ortiz presenta y discute el contexto de la reforma urbana que se inicia con los procesos de descentralización territorial en la década de los ochenta y sus contradicciones con las políticas de orientación progresista incorporadas por la Ley de Desarrollo Territorial -Ley $N^{\circ} 388$ de 1997-, y las implicaciones neoliberales en el mercado inmobiliario; luego, se centra en el análisis y la problematización de las asociaciones público-privadas, promovidas por el nuevo marco jurídico de ordenamiento territorial colombiano, que enmarcan la gestión de los grandes proyectos urbanos. El último artículo, evalúa los discursos planificadores de Bogotá orientados a la conformación de una zona destinada para la innovación tecnológica; sus autores -Chaparro y Aguilar- hacen una revisión de algunos aspectos teóricos sobre la innovación tecnológica y sus relación con el espacio urbano, analizan algunos de los impactos territoriales asociados a este tipo de iniciativas, y finalmente, analizan las contradicciones políticas y territoriales en la incorporación de corredores tecnológicas en la planificación urbana tomando como caso Bogotá.

En síntesis, el libro abre una amplia perspectiva en términos de variables y tendencias metropolitanas: gobernanza metropolitana, morfología, dinámicas demográficas, relaciones regionales y dimensión ambiental que se desarrollan a través de las experiencias en Santiago de Chile, Bogotá, Concepción y Medellín; dentro de estos desarrollos teóricos y empíricos, uno de los aportes más relevantes es la validación de la vigencia del concepto de metrópolis en las estructuras espaciales urbanas contemporáneas a partir de la reelaboración del concepto que transita desde una dominación política y económica dominante durante el siglo XIX, a una lectura de la socialidad en el siglo $X X, y$ un nuevo giro provocado por los procesos de globalización y reestructuración económica con su correlato en el urbanismo como forma de expansión del capitalismo en la escala global; y la consiguiente, ratificación de la importancia contemporánea de estos fenómenos metropolitanos: creciente urbanización y reestructuración económica multiescalar.

Además, se destacan otros aportes del libro: el reconocimiento de la creciente diversidad de las expresiones espaciales o las formas de urbanización en el capitalismo global (Brenner, 2013) como lo comprueban los análisis teórico-empíricos de los casos estudiados; la importancia de la lectura multiescalar de los procesos de urbanización y las implicaciones de cada una de estas escalas; la dimensión cultural y el cosmopolitanismo que hace parte de las nuevas formas urbanas; la necesaria revisión de los procesos de metropolización en América Latina: la periferia expandida, los modos combinados de crecimiento por expansión y densificación o 
CONCEPCIÓN.

verticalización, la importancia creciente del sector inmobiliario y los servicios asociados a la globalización, las implicaciones de los modelos de gestión territorial público-privados, y los desajustes de la institucionalidad y la gobernanza multiescalar, entre otros aspectos de nuestro contexto latinoamericano.

\section{Referencias bibliográficas}

BRENNER, N. Tesis sobre la urbanización planetaria. Nueva Sociedad, 2013, № 243, p. 38-66.

HIDALGO, R.; BORSDORF, A. y ZUNINO, R. Las dos caras de la expansión residencial en la periferia metropolitana de Santiago de Chile: precariópolis estatal y privatópolis inmobiliaria. En: HIDALGO, R. y PEREIRA, P. (editores). Producción inmobiliaria y reestructuración metropolitana en América Latina. Santiago de Chile: Pontificia Universidad Católica de Chile, Facultad de Arquitectura y Urbanismo - Universidad de São Paulo (FAUSP), 2008, p. 167-195.
SÁNCHEZ, R. La debilidad de la gestión del riesgo en los centros urbanos: el caso del Área Metropolitana de Santiago de Chile. Revista de Geografía Norte Grande, 2010, Nº 47, p. 19-36. 